\title{
Correction to: Molecular characterization of Globodera pallida found in Japan using ribosomal DNA and mitochondrial cytochrome $b$ gene sequences
}

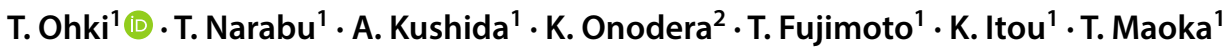

Published online: 3 November 2019

(c) The Phytopathological Society of Japan and Springer Japan KK, part of Springer Nature 2019

\section{Correction to: \\ Journal of General Plant Pathology (2018) 84:230-236 \\ https://doi.org/10.1007/s10327-018-0776-5}

The authors would like to correct the errors in the publication of the original article.

The first eight sentences under the subheading "PCR, cloning, and sequencing" in the "Materials and methods" section contained errors in the primer sequences and in the one of the reference citations.

The sentences should read as:

"The ribosomal DNA sequences (rDNA) including two ITS and 5.8S ribosomal RNA were amplified using the primers of Ferris et al. (1993): forward (CGTAACAAGGTAGCT GTAG) and reverse (TCCTCCGCTAAATGATATG). Partial sequences of the mitochondrial cytochrome $b$ gene $(c y t b)$ were amplified using primers INRAcytbL (GGGTGTGGC CTTGTTATTTC) and INRAcytbR (ACCAGCTAAAAC CCCATCCT) (Picard et al. 2007)."

The below reference should be included in the reference list.

\section{Reference}

Ferris VR, Ferris JM, Faghihi J (1993) Variation in spacer ribosomal DNA in some cyst-forming species of plant parasitic nematodes. Fundam Appl Nematol 16:177-184

Publisher's Note Springer Nature remains neutral with regard to jurisdictional claims in published maps and institutional affiliations.

The original article can be found online at https://doi.org/10.1007/ s10327-018-0776-5.

\section{T. Ohki}

take2001@affrc.go.jp

1 Division of Agro-environmental Research, Hokkaido Agricultural Research Center, National Agriculture and Food Research Organization, 1 Hitsujigaoka, Toyohira, Sapporo, Hokkaido 062-8555, Japan

2 Agricultural Production Environment Group, Kitami Agricultural Experiment Station, Hokkaido Research Organization, Yayoi, Kunneppu, Hokkaido 099-1496, Japan 